\title{
Trauma medular em região toracolombar em gato: Relato de caso
}

\author{
Marlos Melo Duarte ${ }^{1}$, Paulo Victor Garrêto Rodrigues dos Santos ${ }^{1}$, Sérgio Diego Passos \\ Costa $^{1}$, Kellen Matuzzy Silva de Melo ${ }^{1}$, Thays Garreto Rodrigues dos Santos ${ }^{2}$, Francisco \\ Lima Silva ${ }^{3}$ \\ ${ }^{I}$ Médico Veterinário Residente do Hospital Veterinário da Universidade Federal de Piauí (UFPI). Teresina, Piauí, Brasil. E- \\ mail: victorgarreto@ hotmail.com \\ ${ }^{2}$ Aluna do curso de Medicina Veterinária da Universidade Federal de Piauí (UFPI). Teresina, Piauí, Brasil. \\ ${ }^{3}$ Prof. - Departamento de Clínica e Cirurgia Veterinária da Universidade Federal de Piauí (UFPI). Teresina, Piauí, Brasil.
}

RESUMO. Este relato de caso mostra a preocupação com os traumas medulares que os gatos sofrem, a questão da procura por um veterinário tardiamente, o que pode prejudicar o tratamento com sucesso. Relata o caso de um trauma toracolombar, considerado um dos traumas medulares mais difíceis de reversão, onde o felino foi submetido ao tratamento cirúrgico e obteve sucesso na resposta, mesmo sendo uma lesão um pouco tardia.

Palavras chaves: lesão medular; coluna vertebral; vértebra lombar.

\section{Spinal cord injury in thoracolumbar region in cat: Case report}

\begin{abstract}
This case report shows a concern with the medullary traumas that cats suffer, the issue of looking for a veterinarian late, which could affect the successful treatment. We report the case of hum thoracolumbar trauma, considered one of medullary trauma more difficult to reverse, where the feline was submitted to surgical treatment and was successful response, even with a slightly late injury.
\end{abstract}

Keywords: spinal cord injury; Spine; lumbar vertebra.

\section{Introdução}

$\mathrm{Na}$ medicina veterinária, principalmente em cães e gatos, é comum observar que as lesões de coluna são ocasionadas tanto pela forma endógena como pela forma exógena (Bagley et al., 1999). Lesões causadas por agentes endógenos relacionam-se com protrusões ou extrusões de disco intervertebral, assim como também com fraturas patológicas nessa região, ou alterações morfológicas de caráter genético. Quando a origem é exógena têm como principais causadores os traumas ocasionados por automóveis, mordedura de outros animais, quedas e até mesmo lesões por projéteis (Platt et al., 2005).

Nos animais, como também nos seres humanos, uma lesão medular pode trazer bastantes danos, inclusive a inviabilidade permanente dos membros, tornando o seu tratamento muito difícil para as medicinas, já que o sistema nervoso central não tem capacidade de regenerar seus neurônios, e também por não haver um consenso sobre o tratamento ideal (Blight, 2000; Olby \& Jeferry, 2003). Segundo estudos, cerca de 12,8\% dos cães e gatos que são atendidos na urgência chegam com reclamações em decorrência de algum trauma, sendo os gatos mais frequentes que os cães, considerado até o dobro de casos (Kolata, 1981; Rabelo, 2008). Esse número alto de casos em felinos pode ter origem na facilidade que os gatos têm de acesso à rua (Adamantos \& Corr, 2007). Por possuírem menos massa corporal e menor peso em relação aos cães, os gatos podem sofrer traumas mais graves (Kolata, 1981).

A abordagem primária em pacientes com trauma deve se iniciar pela avaliação do sistema cardiovascular e respiratório de forma rápida e precisa. Os sistemas neurológico e renal devem ser avaliados de forma bem detalhada, principalmente em gatos, onde os danos podem levar ao óbito do animal (Tello, 2008). O exame neurológico deve ser feito com muito cuidado para não mexer exacerbadamente no animal, por conta do não conhecimento de possíveis fraturas na coluna vertebral, e até mesmo instabilidades. O nível de consciência, a frequência e amplitude respiratória, 
a postura, reflexos do tronco e membros devem ser avaliados detalhadamente, sempre tomando cuidado também com o pescoço do animal (Arias, 2007).

\section{Relato de caso}

Um felino, fêmea, sem raça definida, com nove meses, pesando $2,45 \mathrm{~kg}$, foi atendido no Hospital Veterinário Universitário da Universidade Federal do Piauí (HVU-UFPI), sendo relatado pelo tutor na anamnese que o animal tinha caído do $4^{\circ}$ andar do apartamento, sendo que o mesmo apresentava dor intensa, vocalização e não movimentava os membros pélvicos. A Tutora relatou também que o animal não foi levado no mesmo momento do acontecido para cuidados médicos, não apresentava nenhuma enfermidade antes do acontecido e que o mesmo gostava muito de andar na varanda do apartamento, a mesma assumiu o erro por não ter colocado uma proteção na varanda.

No exame clínico foi observado na mucosa oral e ocular normocoradas, tempo de preenchimento capilar (TPC) menor que 2 segundos, paciente apresentava uma leve desidratação, linfonodos normais, taquipnéia e frequência cardíaca normal, pouca sensibilidade em membros posteriores (sem dor superficial ou profunda), possuía sensibilidade em cauda, reflexos pupilares normais, vesícula urinária repleta, paciente sem sinal de choque. A princípio foram solicitados os seguintes exames complementares: Exame hematológico completo, radiografia simples de coluna e posteriormente exames bioquímicos de sangue e também o exame de ultrassonografia.
$\mathrm{O}$ animal foi internado e imobilizado para evitar traumas secundários, logo foi feita a administração de fluídos (ringer com lactato), anti-inflamatório não esteroidal (cetoprofeno), protetor de mucosa gástrica (ranitidina), analgésicos (tramadol e fentanil), antibiótico (cefazolina), complexo de vitamina B. Após os resultados dos exames de imagem (Figura 1), foram encontradas fratura por compressão e subluxação vertebral em região toracolombar (fratura em L1 e possível compressão entre os seguimentos T13, L1 e L2), posteriormente foi realizada a cirurgia onde foi aplicada a distração, descompressão através de laminectomia, e fixação permanente com pino de Steinmann duplo, número 1,5, em cada vértebra dois pinos em T13 e dois pinos em L2 (Figura 3).

Durante a cirurgia foi encontrado um fragmento do pedículo proximal da vértebra L2 comprimindo a raiz principal da mesma do lado direito (Figura 2) e a mesma foi removida com cuidado. No último passo foi colocado o cimento ósseo para finalizar a fixação permanente (Figura 4 e 5), e finalmente as suturas de aproximação das fáscias musculares, subcutâneo e pele. Após 24 horas da cirurgia, o paciente já apresentava sensibilidade superficial e um melhor controle da micção. Foi avaliado seu estado geral e constatado melhora significativa tanto na sensibilidade dos membros pélvicos, como na micção, o mesmo foi encaminhado para fisioterapia com todas as recomendações devidas passadas para os proprietários. Após 13 dias de internação o paciente teve alta.

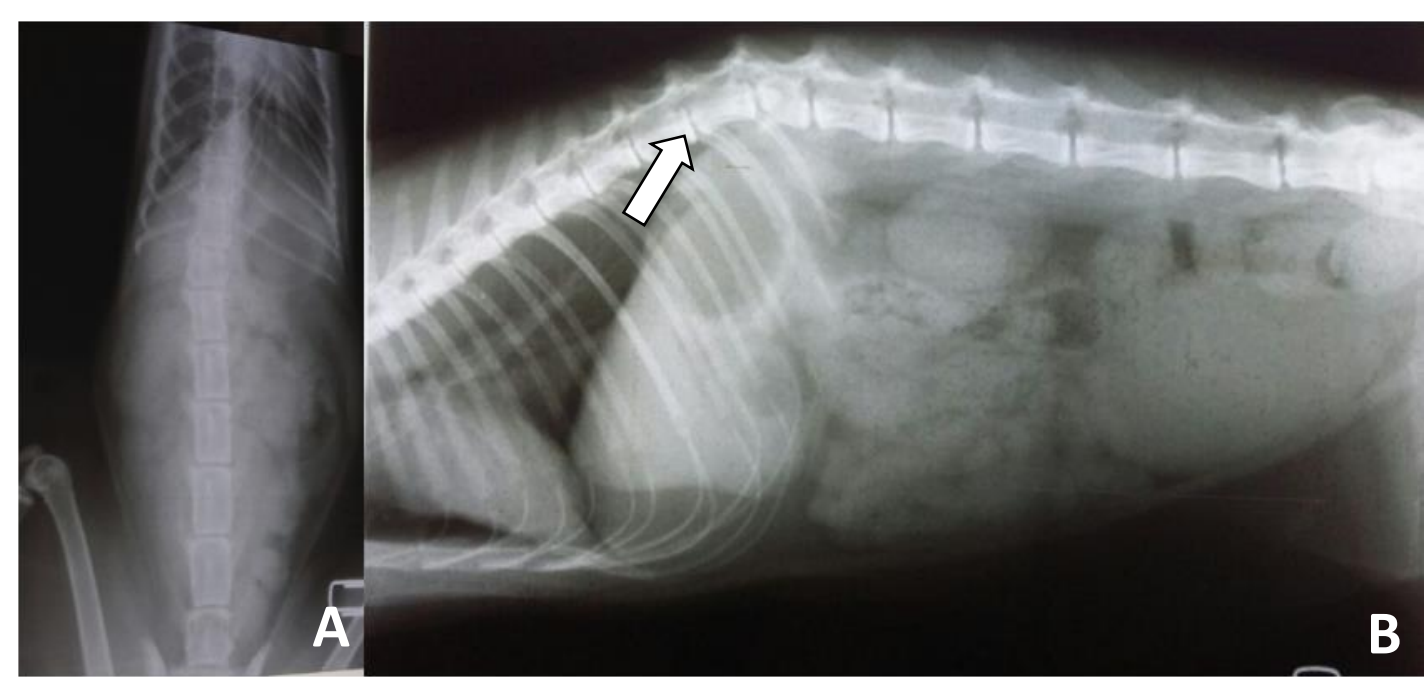

Figura 1: A. Posição dorsoventral. B. Posição latero-lateral. Fratura em L1 (seta). Fonte: Arquivo Pessoal 


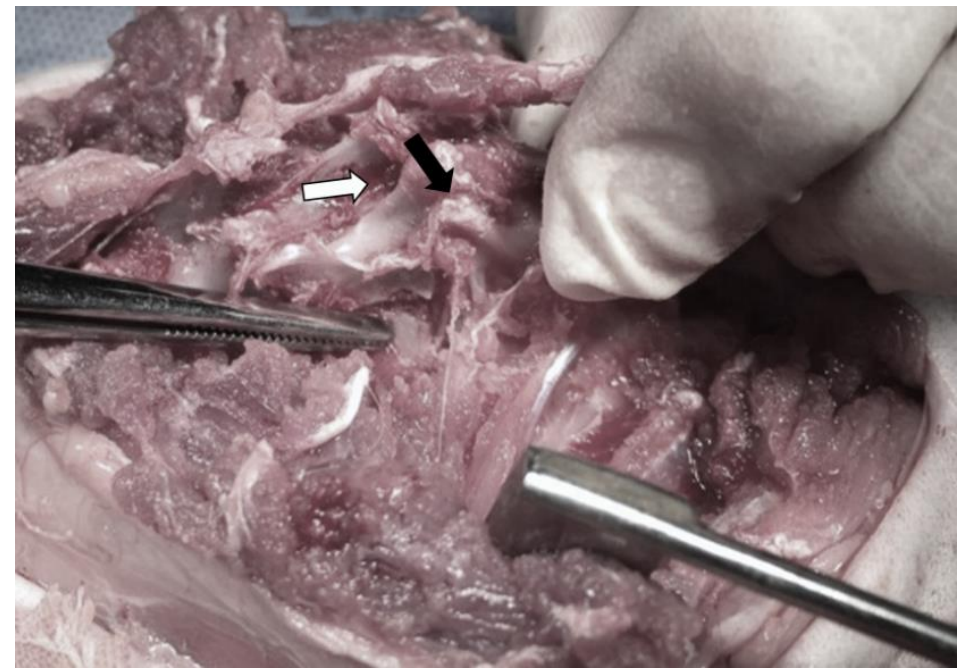

Figura 2. Fragmento do pedículo fraturado (seta branca) comprimindo a raiz principal da vértebra (seta preta). Fonte: Arquivo Pessoal

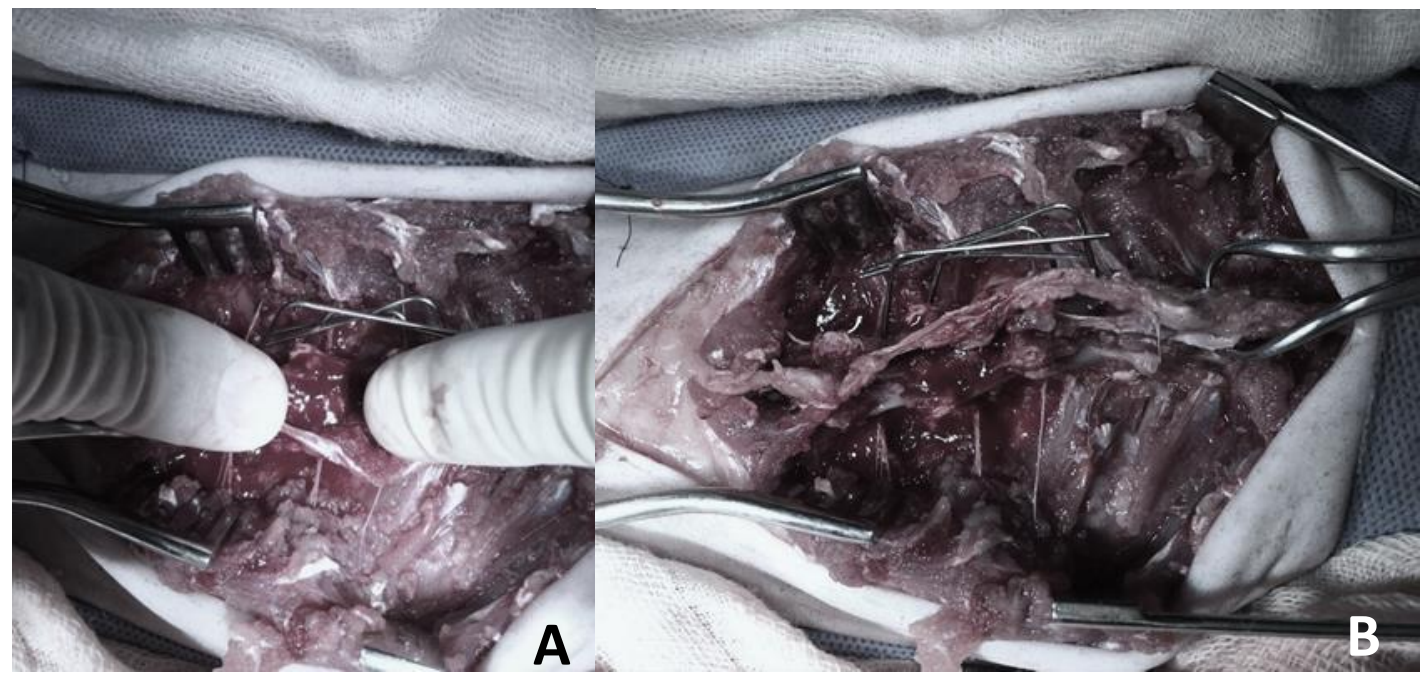

Figura 3. A. Fixação unilateral permanente com pino de Steinmann duplo. B. Dois pinos em T13 e dois pinos em L2. Fonte: Arquivo Pessoal

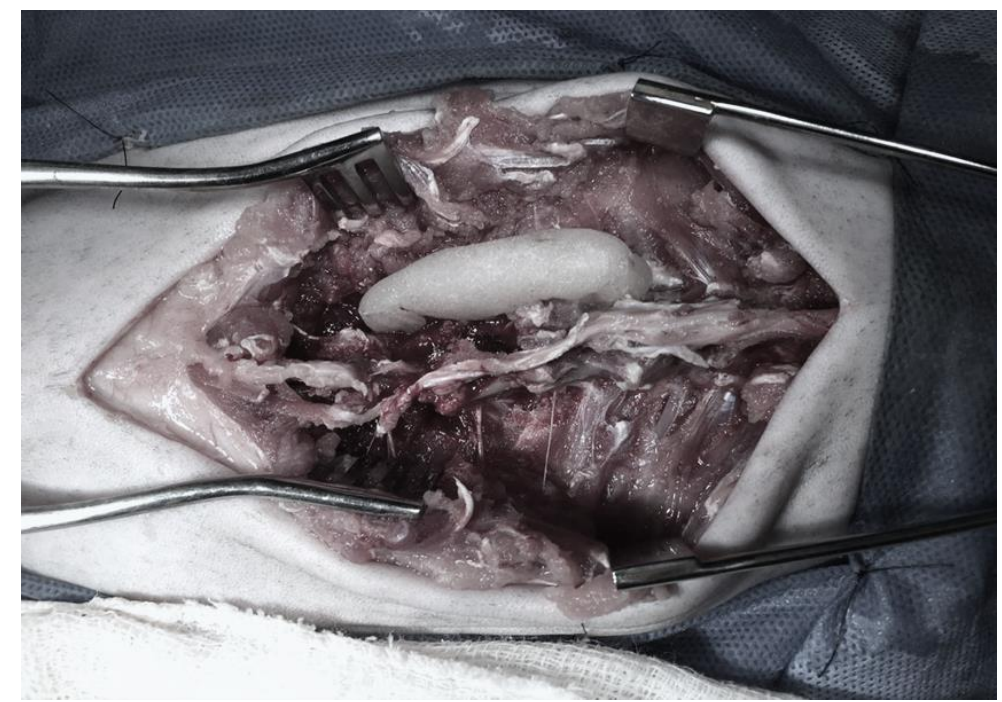

Figura 4: Cimento ósseo para finalizar a fixação permanente. Fonte: Arquivo Pessoal 


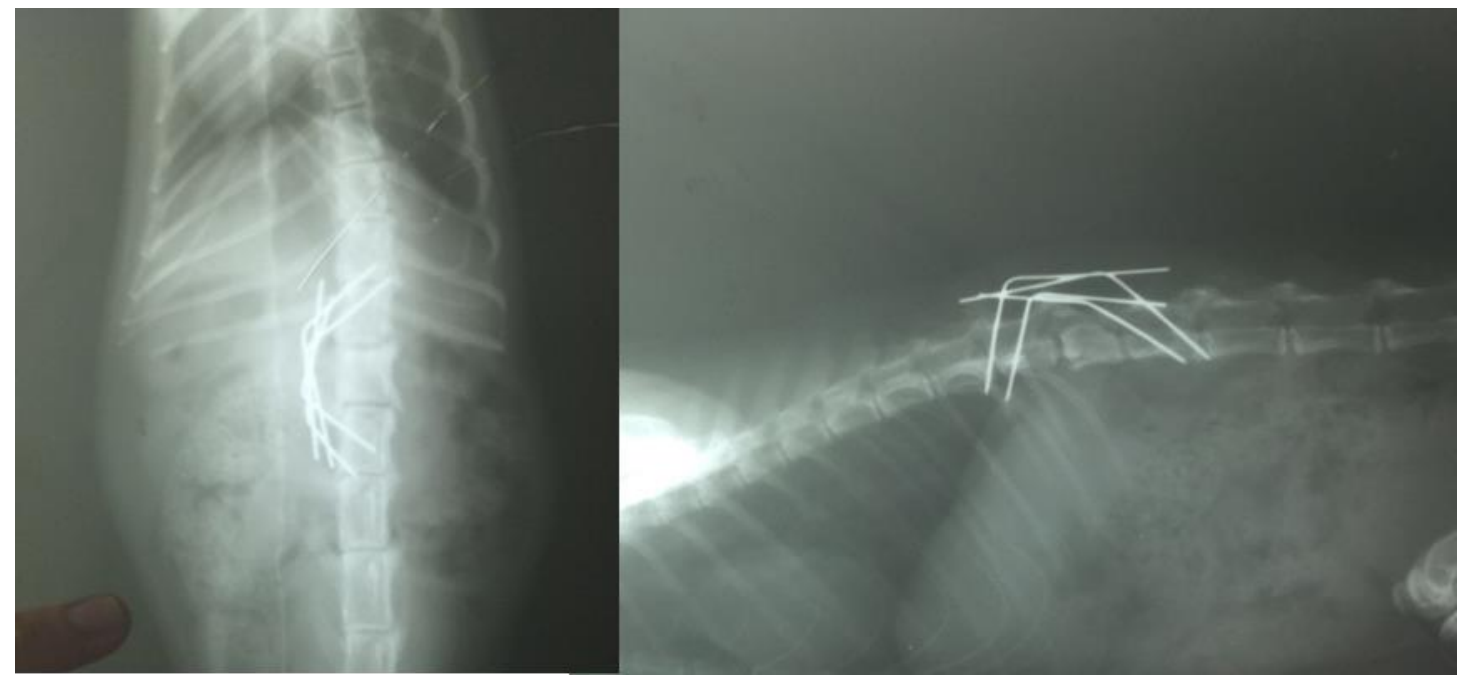

Figura 5. Radiografia no pós-cirúrgico mostrando o resultado da técnica. Fonte: Arquivo Pessoal

\section{Resultados e Discussão}

No exame físico, os resultados encontrados no paciente com lesão na medula foram bem específicos, como perda dos movimentos dos membros, perda do controle de micção, ausência de dor ao estimular os membros. O local da lesão pode determinar o grau do prognóstico, considerando assim o trabalho de Mendes \& Arias (2012) que afirmaram que 57\% dos animais com lesão da medula na porção toracolombar vem a óbito, o paciente aqui citado obteve grande sucesso no tratamento. Mendes \& Arias (2012) também afirmaram que a presença de sensibilidade dolorosa profunda afeta a recuperação, já Bagley et al. (1999) falaram que é necessário a presença de sensibilidade dolorosa profunda para que o prognóstico seja favorável em lesões de medula na região toracolombar, como no caso citado, onde o animal possuía pouca sensibilidade dolorosa nos membros posteriores, porém a região da cauda já mostrava uma sensibilidade maior. Bergman et al. (2000) confirmam que apenas uma lesão muito severa na medula espinhal pode reduzir essa sensibilidade dolorosa, o que aconteceu também no caso, onde o animal sofreu uma queda da altura de 4 andares. Jorge (2009) cita que o prognóstico se torna ruim quando existe uma percepção dolorosa perdida, principalmente no caso de dor profunda, mostrando assim que o paciente desse caso corria risco, porém com a técnica bem realizada e todos os parâmetros estabilizados, houve uma chance de recuperação bem sucedida.
$\mathrm{Na}$ avaliação da imagem radiográfica só foi possível observar o grau da lesão na coluna vertebral, lesões na medula não foram observadas, o que nesse caso foi feito com o exame neurológico, de acordo com Araújo et al. (2009). Se a lesão tivesse promovido um deslocamento de 30 a $100 \%$ da coluna vertebral, o animal não teria possibilidades de voltar a ter o movimento de seus membros, o que não ocorreu nesse caso. $\mathrm{Na}$ ultrassonografia não apareceu nada digno de nota. Mesmo o tutor levando o animal dias depois do acidente, não houve muita complicação no trans e pós-cirúrgico, porém o animal com um trauma medular deve ser trazido ao atendimento o mais precocemente, para ajudar numa melhor avaliação, estabilizar a volemia, imobilizar o animal para evitar lesões adicionais pela tentativa de movimentar-se e proceder tratamento a lesões concomitantes, corroborando com Bergman et al. (2000).

As lesões primárias como vértebras deslocadas, coluna instável e hematomas locais causam maior compressão medular, o que provoca um déficit na perfusão do tecido nervoso, causando inflamação, liberação de radicais livres e destruição do tecido, por conta disso o animal após a avaliação neurológica foi imobilizado, colaborando com as informações de Olby (2010).

O tratamento para lesão medular segue duas vertentes, ou o clínico opta pelo tratamento conservador ou pelo tratamento cirúrgico. No presente trabalho o tratamento cirúrgico se fez necessário por causa da instabilidade que a fratura 
promovia na coluna vertebral e por conta também da compressão na medula espinhal, entrando em consenso com Bagley et al. (1999).

\section{Conclusão}

A sensibilidade dolorosa na região dos membros afetados é de suma importância para o prognóstico do trauma medular. Utilizando-se técnicas apropriadas e destreza no momento que o animal chega na urgência/emergência são primordiais para o sucesso no pós-cirúrgico.

\section{Referências Bibliográficas}

Adamanios, S. \& Corr, S. (2007). Emergency care of the cat with multi-trauma. In practice, 29, 388-396.

Araújo, B. M., Arias, M. V. B. \& Tudury, E. A. (2009). Paraplegia aguda com perda da percepção de dor profunda em cães: revisão de literatura. Clínica Veterinária, 81, 70-82.

Arias, M.V.B. (2007) [Online]. Trauma craniano - abordagem e tratamento. Homepage: http://bahrbituricos.blogspot.com/2007/08/tra uma-craniano-abordageme-tratamento.html. Acesso em: 01/07/2016

Bagley, R. S., Harrington, M. L., Silver, G. M., Cambridge, A. J., Connors, R. L. \& Moore, M. P. (1999). Exogenous spinal trauma: clinical assessment and initial management. Veterinary Neurology, 21, 1138- 1143.

Bergman, R., Lanz, O. \& Shell, L. (2000). Acute spinal cord traume: mechanisms and clinical syndromes. Veterinary Medicine, 95, 846-850.

Blight, A.R. (2000). New drugs for spinal trauma. In: American college of veterinary internal medicine forum, 18. Proceedings..., 292-293.

Jorge, S. M. C. (2009). Traumatismos medulares em canídeos. Dissertação de Mestrado Integrado em Medicina Veterinária. Faculdade de Medicina Veterinária, Universidade Técnica de Lisboa. p.85.

Kolata (1981). Patterrns and Incidence of trauma. In: Archibald J., Holt, J. \& Sokolovsky, V.
Management of Trauma in Dogs and Cats. American Veterinary Publications, Inc. Santa Barbara, U.S.A. 11-20.

Mendes \& Arias, M. V. B. (2012). Traumatismo da medula espinhal em cães e gatos: estudo prospectivo de 57 casos. Pesquisa Veterinária Brasileira, 32:1304 1312.

Olby, N. (2010). The pathogenesis and treatment of acute spinal cord injuries in dogs. Veterinary Clinics of North America: Small Animal Practice, 40, 791-807.

Olby, N. \& Jeffery, N. (2003). Pathogenesis of diseases of the central nervous system. In: SLATTER, D. Textbook of Small Animal Surgery. 2.ed. Philadelphia: Cap.77, p.11321147.

Platt, S. R., Abramason, C. J. \& Garosi, L. S. (2005). Administering corticosteroids in neurologic diseases. Compendium on Continuing Education for the Practicing Veterinarian, Princeton, 27, 210-220.

Rabelo, R.C. (2008). Estudio y valor pronóstico de los parámetros relacionados com supervivência en clinica de urgencias de pequeños animales: Estudio Multicéntrico. Tesis Doctoral. Facultad de Veterinaria, Universida de Complutense de Madrid, 256p.

Tello, L.H. (2008). Trauma em pequenos animais. In: Trauma em cães e gatos. $1^{\circ}$ ed. São Paulo: MedVet Livros.

\section{Article History:}

Received 28 July 2016

Accepted 16 August 2016

Available on line 19 September 2016

License information: This is an open-access article distributed under the terms of the Creative Commons Attribution License, which permits unrestricted use, distribution, and reproduction in any medium, provided the original work is properly cite. 